\title{
HARMONIC CAVITIES FOR THE NLC DAMPING RINGS*
}

\author{
S. de Santis", A. Wolski, LBNL, Berkeley, CA 94720, USA
}

\section{Abstract}

To achieve high luminosity, a linear collider needs damping rings to produce beams with very small transverse emittances. In the NLC, design constraints place the Main Damping Rings in a parameter regime where intrabeam scattering (IBS) is likely to be a limitation on the emittance, and hence on the final luminosity. It is possible to mitigate the effects of IBS by lengthening the bunch: this may be achieved by redesigning the lattice with higher momentum compaction, or by use of higher harmonic cavities. Here, we consider the latter approach. We estimate the required bunch lengthening that might be needed, outline some appropriate parameters for the harmonic cavities, and discuss some of the effects that might be introduced or exacerbated by the cavities, such as synchronous phase variation along the bunch train.

\section{THE NLC MAIN DAMPING RINGS}

A lattice design for the NLC Main Damping Rings (MDRs) was produce in April 2001 [1]. Some relevant parameters are given in Table 1. This design met the specifications for damping rate and emittance at low current, but studies of a variety of collective effects motivated a new design [2] with a longer bunch. Among the effects causing concern was intrabeam scattering, which was estimated to increase the extracted horizontal emittance some way above the specified value.

Table 1: Lattice Parameters for the 2001 NLC MDR

\begin{tabular}{|l|l|}
\hline Energy & $1.98 \mathrm{GeV}$ \\
\hline Circumference & $299.792 \mathrm{~m}$ \\
\hline Natural emittance (normalized) & $2.2 \mu \mathrm{m}$ \\
\hline Equilibrium vertical emittance & $0.014 \mu \mathrm{m}$ \\
\hline Mean beta functions (x,y) & $3.6 \mathrm{~m}, 7.1 \mathrm{~m}$ \\
\hline Mean horizontal H functions & $1.9 \mathrm{~mm}$ \\
\hline Expected rms vertical dispersion & $1.5 \mathrm{~mm}$ \\
\hline Transverse damping times (x,y) & $4.8,5.0 \mathrm{~ms}$ \\
\hline Longitudinal damping time & $2.6 \mathrm{~ms}$ \\
\hline Momentum compaction & $2.95 \times 10^{-4}$ \\
\hline Natural bunch length & $3.7 \mathrm{~mm}$ \\
\hline Natural energy spread & $0.091 \%$ \\
\hline Bunch charge & $7.5 \times 10^{9}$ \\
\hline Bunch store time & $25 \mathrm{~ms}$ \\
\hline
\end{tabular}

Before attempting a new lattice design, the possibility of increasing the bunch length using harmonic cavities was considered. Here, we report on the results of the study of the effects of the harmonic cavities, and discuss the reasons for choosing a new lattice as the preferred approach to reducing the severity of the collective effects.

\footnotetext{
*Work supported by the US DOE contract DE-AC03-76SF00098. "SDeSantis@lbl.gov
}

\section{IBS ESTIMATES}

IBS is not generally a significant effect in electron storage rings, since the growth rates are usually much smaller than the radiation damping rates. Observations have been made at the KEK ATF [3] and the ALS at LBNL [4], both operating in regimes where the coupling is very small. There is some uncertainty as to whether the measurements made at the ATF are fully explained by the IBS model $[3,5]$; however, since the issue is presently unresolved, we base our estimates on this model, using approximations that are valid in the relevant regime [6]. Using the method described in [3], we can calculate the evolution of the transverse and longitudinal emittances as functions of time after the injection of the beam into the damping ring, including the effects of IBS and radiation damping.

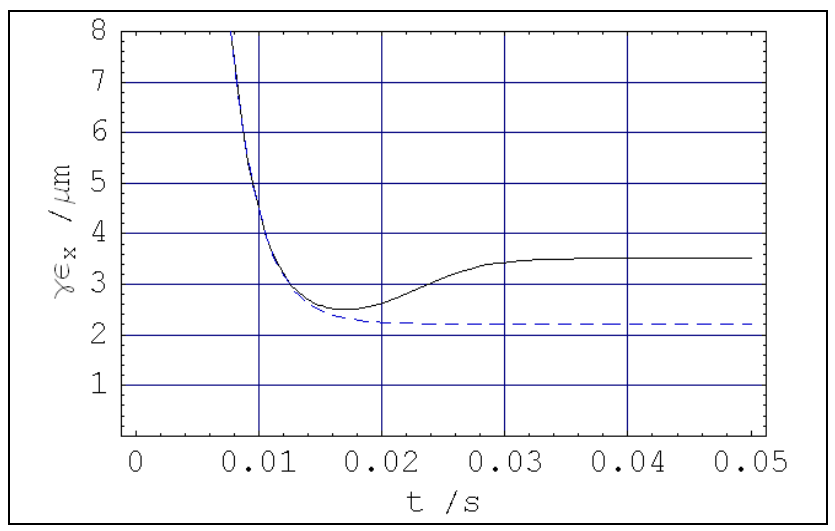

Figure 1: Evolution of horizontal emittance with IBS (solid line) and with radiation only (broken line)

Figure 1 shows the evolution of the horizontal emittance following injection into the ring with a normalized emittance of $150 \mu \mathrm{m}$. IBS also leads to an increase (though less severe) in the vertical and longitudinal emittances. In the horizontal plane, we observe the characteristic minimum associated with IBS, which becomes strong enough to increase significantly the horizontal emittance only when the vertical emittance has damped to a certain value. At equilibrium, the horizontal emittance is increased to $3.51 \mu \mathrm{m}$. The specified extracted emittance is $3.0 \mu \mathrm{m}$.

Increasing the bunch length reduces the charge density and thus reduces the IBS growth rates. However the extracted horizontal emittance is a relatively weak function of the natural bunch length (Figure 2): in order to achieve $3 \mu \mathrm{m}$ extracted emittance, the bunch length needs to be increased to between $4.5 \mathrm{~mm}$ and $5.0 \mathrm{~mm}$, depending on the equilibrium vertical emittance. Note that the equilibrium vertical emittance needs to be reduced close to $0.01 \mu \mathrm{m}$ in order to achieve $0.02 \mu \mathrm{m}$ extracted, with IBS. 


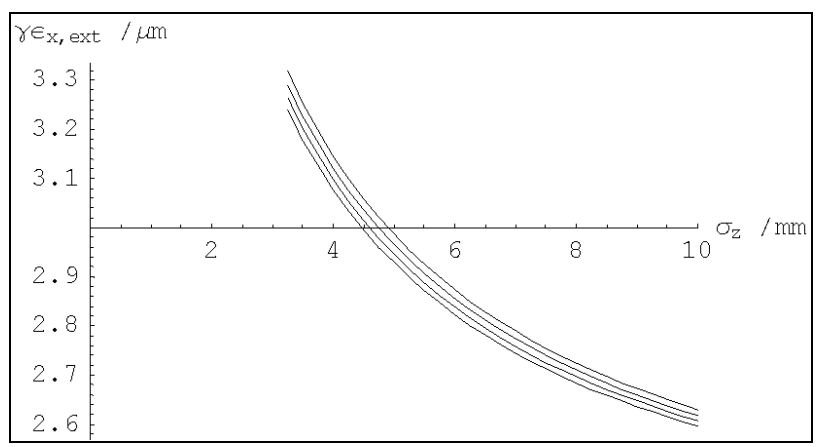

Figure 2: Extracted horizontal emittance as a function of natural bunch length, for equilibrium vertical emittances from $0.013 \mu \mathrm{m}$ to $0.010 \mu \mathrm{m}$ (from left to right).

\section{LENGTHENING THE BUNCH WITH A HIGHER HARMONIC VOLTAGE}

The fundamental theory of a higher harmonic RF system is described in [7]. In our case we are interested in bunch lengthening of around 30\%, which allows the possibility of using a somewhat simplified theory and rather relaxed requirements for the cavity parameters.

Higher harmonic RF systems are already in use, or are being designed, in a number of electron storage rings and have been the object of recent studies [8]. The main problem connected with the use of harmonic cavities, in either passive or active mode, is the transient beam loading induced on the bunch train. The difference in the bunch phases, relative to the RF voltage, can assume very high values, which make obtaining a uniform bunch lengthening difficult. As an additional problem in the NLC damping rings, the systems downstream of the damping ring accept a limited variation (less than $10 \mathrm{~mm}$ ) of the bunch phase at input. It should be noted that simulations indicate a $6 \mathrm{~mm}$ phase variation from just the main RF system.

In first approximation, we can say that the bunch lengthening is proportional to the inverse of the derivative of the total RF voltage. Hence:

$$
\frac{V_{R F}^{\prime}}{V_{T o t}^{\prime}}=\frac{\sigma_{z h}}{\sigma_{z}}
$$

where $\sigma_{z h}$ is the bunch length, when the harmonic voltage $V_{h}(t)$ is applied and

$$
V_{T o t}^{\prime}=V_{R F}+V_{h}
$$

With our parameters, Eq.(3) yields a value for the required peak harmonic voltage of about $80 \mathrm{kV}$.

Common beam loading models are not adequate to describe the effects with harmonic cavities. For this reason we use a simple tracking code, developed by J. Byrd at the Advanced Light Source, in which we compute the transient voltages, induced by the uneven fill, in the main and harmonic RF system. This code is also used for determining the fundamental parameters of the harmonic cavities, based on the target harmonic voltage and the MDR parameters (Table 2). At this first stage, the bunches are treated as point-like charges and it is possible to include the effects of the main RF cavities high-order modes and random non-uniformities in the bunch charges (which turn out finally to be negligible).

Table 2: Parameters for the 2001 NLC MDR RF system

\begin{tabular}{|l|l|}
\hline Main RF frequency & $714 \mathrm{MHz}$ \\
\hline Shunt impedance & $3.744 \mathrm{M} \Omega$ \\
\hline R/Q & 117 \\
\hline Coupling factor & 12.33 \\
\hline Peak RF voltage & $1.07 \mathrm{MV}$ \\
\hline Energy loss/turn & $792 \mathrm{keV}$ \\
\hline DC beam current & $729 \mathrm{~mA}$ \\
\hline Number of bunches & $3 \times 192$ \\
\hline
\end{tabular}

The total voltage (main RF voltage plus harmonic voltage) is then used for calculating the length of each individual bunch.

\section{SIMULATION RESULTS}

Table 3 reports the main parameters for the third harmonic RF system used in the tracking code. These parameters where chosen to obtain a harmonic voltage higher than the $80 \mathrm{kV}$ reported in the previous paragraph: equations 2 and 3 underestimate the required voltage, since they do not include the additional energy loss due to the harmonic cavity and the phase relation between fundamental and harmonic voltage.

Table 3: RF Parameters for the 2001 NLC MDR third harmonic RF system

\begin{tabular}{|l|l|}
\hline Harmonic RF frequency & $2142.4 \mathrm{MHz}$ \\
\hline Shunt impedance & $0.7 \mathrm{M} \Omega$ \\
\hline R/Q & 35 \\
\hline
\end{tabular}

As previously stated, such parameters for the harmonic cavity can be easily obtained with a traditional single cell copper cavity. The harmonic cavity is tuned $0.4 \mathrm{MHz}$ above the third RF harmonic because of stability considerations.

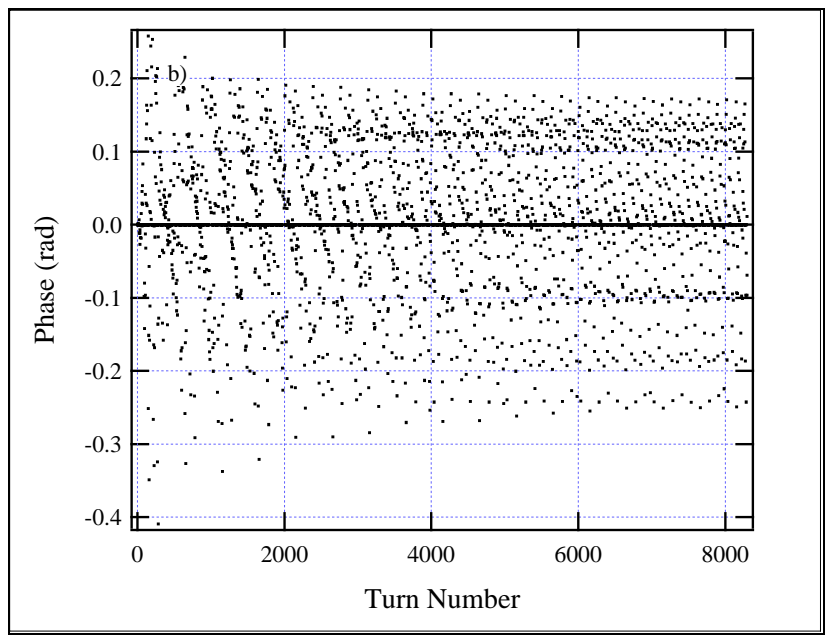

Figure 3: Turn-by-turn phase of bunches in the NLC MDR.

Our simulation code tracks the three trains of 192 bunches each and calculates their phases (referred to the main RF frequency) turn by turn. Figure 3 shows the 
evolution in the bunch phases from an arbitrary initial distribution, for the first 8300 turns, which corresponds to the MDR injection/extraction cycle. It can be seen that the first 3000 turns are enough to damp the bunch phases to their regime values.
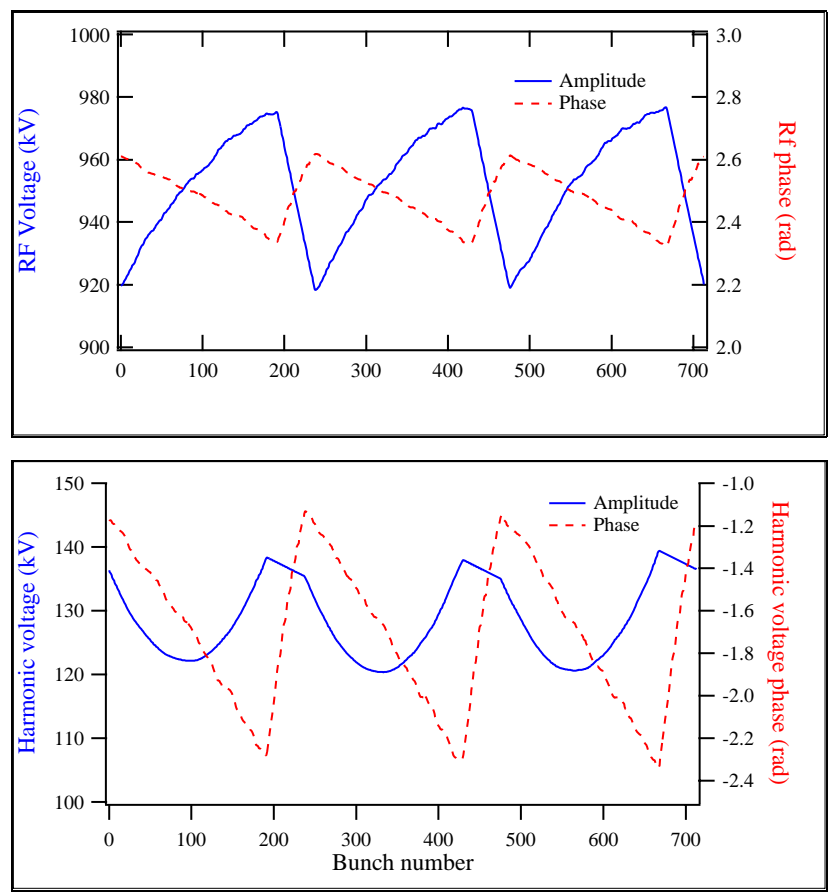

Figure 4: Fundamental (upper) and harmonic (lower) RF voltages and phases.

The fundamental and harmonic RF voltages and phases along the bunch trains are shown in Figure 4. These results include the high-order modes of the main cavities and a $10 \%$ random fluctuation in the bunch charges. The gaps are included in the calculation, since from the point of view of the tracking code, these are simply bunches with zero charge.

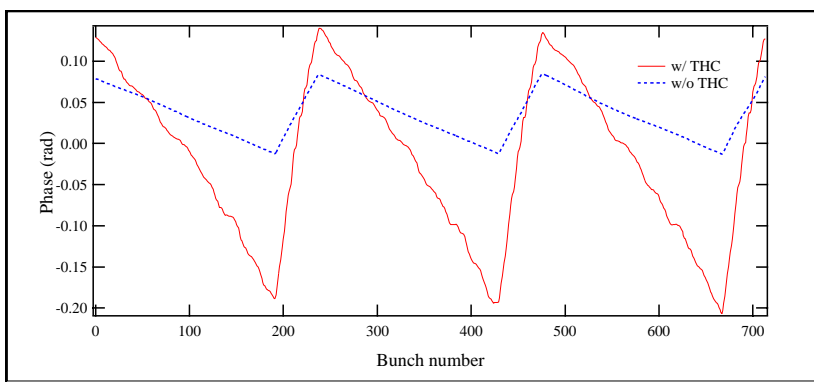

Figure 5: Bunch phase transients with and without the harmonic RF system.

Figure 5 shows the resulting variation of the bunch phases. As a reference, we report also the same variation calculated without the third harmonic cavity, which turns out to be substantially smaller. The phase transient with the harmonic cavity is far larger than the maximum specified for the bunch compressor.

As a final check, the new bunch lengths, after the harmonic voltage is applied, are calculated and reported in
Fig.6, showing that the applied voltage is indeed necessary for obtaining the $30 \%$ required lengthening.

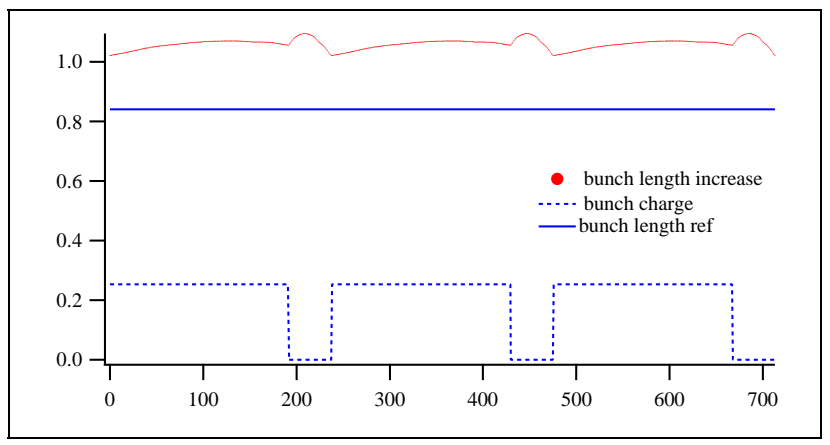

Figure 6: Bunch lengthening.

\section{CONCLUSIONS}

Our calculations show that a simple higher harmonic RF system could be easily designed to obtain the bunch lengthening required to overcome the IBS related emittance increase in the NLC MDR. Because of the intrinsic characteristics of such systems, the effect on the bunch phase distribution would be substantially larger than the maximum specified for the bunch compressor.

Though it might be possible to design a more complex system (with energy storage cavities, for instance) able to reduce the phase transient, it was decided to investigate a lattice redesign, which could give a longer bunch without the need for a higher harmonic RF system.

\section{ACKNOWLEDGMENT}

The authors wish to thank Dr. J. M. Byrd for his invaluable advice.

\section{REFERENCES}

[1] A. Wolski and J. Corlett, "The Next Linear Collider Damping Ring Lattices”, PAC'01, p.3795.

[2] A. Wolski et al, "A Lattice with Larger Momentum Compaction for the NLC Main Damping Rings", these proceedings.

[3] K.L.F. Bane et al, "Intrabeam Scattering Analysis of ATF Beam Measurements”, SLAC-PUB-8875, 2001.

[4] J. Corlett et al, "Measurements of Intrabeam Scattering at Low Emittance in the Advanced Light Source", HEACC'01.

[5] J.D. Bjorken and S.K. Mtingwa, "Intrabeam Scattering", Particle Accelerators 13 p.115, 1983.

[6] T.O. Raubenheimer, "The Generation and Acceleration of Low Emittance Flat Beams for Future Linear Colliders", SLAC report 387, 1991.

[7] J.M. Byrd, et al. "Design of a Higher Harmonic RF System for the Advanced Light Source", Nucl. Instr. Meth. A 439, 15, 2000.

[8] J.M. Byrd, et al. "Transient Beam loading Effects in Harmonic RF Systems for light Sources”, Phys. Rev. ST-AB 5, 092001, 2002. 